\title{
Methods for Reliable Teleportation
}

\author{
Lev Vaidman and Nadav Yoran \\ School of Physics and Astronomy, Raymond and Beverly Sackler Faculty of Exact Sciences, \\ Tel-Aviv University, Tel-Aviv 69978, Israel.
}

()

\begin{abstract}
Recent experimental results and proposals towards implementation of quantum teleportation are discussed. It is proved that reliable (theoretically, $100 \%$ probability of success) teleportation cannot be achieved using the methods applied in recent experiments, i.e., without quantum systems interacting one with the other. Teleportation proposal involving atoms and electro-magnetic cavities are reviewed and the most feasible methods are described. In particular, the language of nonlocal measurements has been applied which has also been used for presenting a method for teleportation of quantum states of systems with continuous variables.
\end{abstract}

\section{INTRODUCTION}

Teleportation is "... apparently instantaneous transportation of persons etc., across space by advanced technological means" [1]. Recently, Bennett, Brassard, Crepeau, Jozsa, Peres, and Wootters (BBCJPW) [2] proposed a gedanken experiment which they called "quantum teleportation". Many proposals were suggested and a few real experiments [3,5,4] were performed inspired by the BBCJPW work. However, an experiment, demonstrating a reliable (i.e., $100 \%$ successful, given ideal devices) teleportation of an unknown state of an external quantum system has not been performed yet. In this paper we prove that the methods used in the current experiments cannot lead to reliable teleportation and discuss other proposals for teleportation.

Quantum teleportation transports the quantum state of a system and/or its correlations to another system. The state is disintegrated in one place and a perfect replica appears at a distant site. The state or its complete description is never located between the two sites during the transportation. The teleportation procedure, apart from quantum channels (prepared in advanced), requires telegraphing surprisingly small amount of information between the two sites. All these properties justify the name "teleportation" for the BBCJPW procedure. Note that telegraphing classical information cannot be instantaneous. Therefore, teleportation cannot be instantaneous too. This, however, is not surprising, since quantum states can carry information and special relativity does not allow instantaneous transmission of signals.

The organization of this paper is as follows. Section II reviews the original teleportation method. Section III is devoted to the proof that without interaction between "quantum" objects the measurement of the nondegenerate Bell operator, which is the core of the original proposal, cannot be performed. Section [V] analyzes teleportation methods which use quantum-quantum interactions, in particular, a method based on nonlocal measurements [6]. In Section $\mathrm{M}$ we discuss arguably a more promising experimental proposals for reliable teleporta- tion including implementation of "crossed" nonlocal measurements scheme for for teleportation of quantum states of atoms. In Section VI we discuss proposals for teleportation of quantum states of continuous variables. Section VII presents a discussion which concludes the paper.

\section{THE ORIGINAL BBCJPW TELEPORTATION PROCEDURE}

Let us start with the analysis of teleportation of a quantum state of a two-level system. The states under discussion are: spin states of a spin- $\frac{1}{2}$ particle, $|\uparrow\rangle$ and $|\downarrow\rangle$, polarization states of a photon, $|\leftrightarrow\rangle$ and $|\uparrow\rangle$, the states of a photon in a two-arm interferometer, $|a\rangle$ and $|b\rangle$, ground and excited state of an atom (or ion), $|g\rangle$ and $|e\rangle$, photon number states of a microwave cavity, $|0\rangle$ and $|1\rangle$. Mathematically, there is no difference between the analysis of various two-level systems. For the present analysis, following the tradition, we will use spin states, in spite of the fact that this is the only system from the list above for which there is no proposal for a real experiment.

The original BBCJPW teleportation procedure consist of three main stages:

i) Preparation of an EPR pair

$$
|E P R\rangle=\frac{1}{\sqrt{2}}(|\uparrow\rangle|\downarrow\rangle-|\downarrow\rangle|\uparrow\rangle) .
$$

ii) Bell-operator measurement performed on the "input" particle and one particle of the EPR pair. Bell operator has the following eigenstates:

$$
\begin{aligned}
& \left|\Psi_{-}\right\rangle=\frac{1}{\sqrt{2}}(|\uparrow\rangle|\downarrow\rangle-|\downarrow\rangle|\uparrow\rangle), \\
& \left|\Psi_{+}\right\rangle=\frac{1}{\sqrt{2}}(|\uparrow\rangle|\downarrow\rangle+|\downarrow\rangle|\uparrow\rangle), \\
& \left|\Phi_{-}\right\rangle=\frac{1}{\sqrt{2}}(|\uparrow\rangle|\uparrow\rangle-|\downarrow\rangle|\downarrow\rangle), \\
& \left|\Phi_{+}\right\rangle=\frac{1}{\sqrt{2}}(|\uparrow\rangle|\uparrow\rangle+|\downarrow\rangle|\downarrow\rangle) .
\end{aligned}
$$


iii) Transmission of the outcome of the Bell measurement and appropriate unitary operation on the second particle of the EPR pair (the "output" particle). The possible operations are: nothing in the case of $\left|\Psi_{-}\right\rangle$and $\pi$ rotations around $\hat{x}, \hat{y}$, and $\hat{z}$ axes for the there other outcomes.

Completing (i)-(iii) ensures transportation of the pure state of the input particle to the state of the output particle. It also ensures transportation of correlations: if the input particle were correlated to other systems, then the output particle ends up correlated to these systems in the same way.

Unitary operations can be performed more or less effectively on all systems. (The operations on quantum states of a microwave cavity can be performed indirectly through a manipulation of atomic state and the interchange of the states of the atom and the cavity.) Preparation of the EPR pair is also achievable (with various levels of difficulty) for all systems. The main difficulty is performing the Bell measurement.

\section{BELL-OPERATOR MEASUREMENT WITHOUT INTERACTION BETWEEN QUANTUM SYSTEMS}

We shall prove here that it is impossible to perform complete (nondegenerate) Bell-operator measurement without using interaction between quantum systems. We allow any unitary transformation of single particle states and we are allowed to perform any local single-particle measurement.

According to the standard (von Neumann) approach, the measurement procedure can be divided into two stages: the unitary linear evolution and local detection. There are four distinct (orthogonal) single-particle states which are involved in the definition of the Bell states: there are two channels, and a two-level system enters into each channel. We name the channels left $(\mathrm{L})$ and right $(\mathrm{R})$ corresponding to the way the Bell states (2) are written, i.e., in the explicit notation, $\left|\Psi_{-}\right\rangle=(1 / \sqrt{2})\left(|\uparrow\rangle_{L}|\downarrow\rangle_{R}-|\downarrow\rangle_{L}|\uparrow\rangle_{R}\right)$. The general form of the unitary linear evolution of the teleportation procedure for the four states can be written in the following form:

$$
\begin{aligned}
|\uparrow\rangle_{L} & \rightarrow \sum a_{i}|i\rangle, \\
|\downarrow\rangle_{L} & \rightarrow \sum b_{i}|i\rangle, \\
|\uparrow\rangle_{R} & \rightarrow \sum c_{i}|i\rangle, \\
|\downarrow\rangle_{R} & \rightarrow \sum d_{i}|i\rangle,
\end{aligned}
$$

where $\{|i\rangle\}$ is a set of orthogonal single-particle local states. The "linearity" means that the evolution of the particle in one channel is independent on the state of the particle in the other channel and therefore Eq. (3) is enough to define the evolution of the Bell states:

$$
\begin{aligned}
\left|\Psi_{-}\right\rangle & \rightarrow \sum_{i, j} \alpha_{i j}|i\rangle|j\rangle, \\
\left|\Psi_{+}\right\rangle & \rightarrow \sum_{i, j} \beta_{i j}|i\rangle|j\rangle, \\
\left|\Phi_{-}\right\rangle & \rightarrow \sum_{i, j} \gamma_{i j}|i\rangle|j\rangle, \\
\left|\Phi_{+}\right\rangle & \rightarrow \sum_{i, j} \delta_{i j}|i\rangle|j\rangle .
\end{aligned}
$$

In the right hand side the sum is on all different pairs $\{i, j\}$ and the order is irrelevant. States of distinguishable particles correspond to different $|i\rangle$ s. If the particles are identical, $|i\rangle|j\rangle$ signifies properly symmetrized states $(1 / \sqrt{2})\left(|i\rangle_{1}|j\rangle_{2} \pm|j\rangle_{1}|i\rangle_{2}\right)$.

We assume that we have only local detectors, therefore, only the product states $|i\rangle|j\rangle$ (and not their superpositions) can be detected. Measurability of the nondegenerate Bell operator means that there is at least one nonzero coefficient of every kind $\alpha_{i j}, \beta_{i j}, \gamma_{i j}, \delta_{i j}$ and if, for a certain $i, j$, it is not zero, then all others are zero.

If the particles entering the two channels are not identical, then there are strong restrictions on the unitary evolution (3) which follows from the fact that the particles do not change their identity:

$$
\begin{array}{r}
a_{i} \neq 0=>\quad c_{i}=d_{i}=0 \\
b_{i} \neq 0=>c_{i}=d_{i}=0 \\
c_{i} \neq 0=>a_{i}=b_{i}=0 \\
d_{i} \neq 0=>a_{i}=b_{i}=0
\end{array}
$$

The equations for coefficients of the decomposition of the Bell states after the unitary evolution are as follows.

For $i=j$ we have

$$
\begin{aligned}
\alpha_{i i} & =a_{i} d_{i}-b_{i} c_{i}, \\
\beta_{i i} & =a_{i} d_{i}+b_{i} c_{i}, \\
\gamma_{i i} & =a_{i} c_{i}-b_{i} d_{i}, \\
\delta_{i i} & =a_{i} c_{i}+b_{i} d_{i},
\end{aligned}
$$

but from (5) we immediately obtain

$$
\alpha_{i i}=\beta_{i i}=\gamma_{i i}=\delta_{i i}=0 .
$$

For $i \neq j$ we have

$$
\begin{aligned}
& \alpha_{i j}=a_{i} d_{j}+a_{j} d_{i}-\left(b_{i} c_{j}+b_{j} c_{i}\right), \\
& \beta_{i j}=a_{i} d_{j}+a_{j} d_{i}+b_{i} c_{j}+b_{j} c_{i}, \\
& \gamma_{i j}=a_{i} c_{j}+a_{j} c_{i}-\left(b_{i} d_{j}+b_{j} d_{i}\right), \\
& \delta_{i j}=a_{i} c_{j}+a_{j} c_{i}+b_{i} d_{j}+b_{j} d_{i} .
\end{aligned}
$$

Let us assume that $a_{i} \neq 0$ and $\alpha_{i j} \neq 0$. Then, taking into account (5) and the requirement of the measurability of the Bell operator, we obtain 


$$
\begin{gathered}
\alpha_{i j}=a_{i} d_{j}-b_{i} c_{j} \neq 0, \\
\beta_{i j}=a_{i} d_{j}+b_{i} c_{j}=0, \\
\gamma_{i j}=a_{i} c_{j}-b_{i} d_{j}=0, \\
\delta_{i j}=a_{i} c_{j}+b_{i} d_{j}=0 .
\end{gathered}
$$

However, we can immediately see that this set of equations does not have a solution. Since the equation are essentially symmetric relative to the various coefficients, we get the same result while choosing other nonzero coefficients. Therefore, we have proved the statement for distinguishable particles.

In fact, the proof yields more than the unmeasurability of the nondegenerate Bell operator. We have proved that even a degenerate Bell states operator measurement which separates just one Bell state is impossible. Note, however, that degenerate Bell-state operator measurement which distinguishes one pair of Bell states from the other pair is possible. For example, measurements of the $\sigma_{z 1}$ and $\sigma_{z 2}$ distinguishes between the pair of states $|\Psi\rangle$ (for both of which the two outcomes are different) and the pair of states $|\Phi\rangle$ (for which the two outcomes are identical). Obviously, these are demolition measurements and we cannot continue to measure the particles and specify all the Bell states.

Let us turn to the analysis of Bell measurement on identical particles starting with bosons [7]. Taking into account symmetrization, we obtain the equations (6) and (8) for the coefficients for different $i, j$ also for this case, except for the overall factor $1 / \sqrt{2}$ in equations (8) (we remind that $|i\rangle|j\rangle$ means $\left.(1 / \sqrt{2})\left(|i\rangle_{1}|j\rangle_{2}+|j\rangle_{1}|i\rangle_{2}\right)\right)$. For bosons, however, we have no restrictions (5) and therefore, we cannot claim immediately that Eq. (7) holds. The measurability of the nondegenerate Bell operator requires that for any given $i$ at least three out of the coefficients $\alpha_{i i}, \beta_{i i}, \gamma_{i i}, \delta_{i i}$ are zero. From equations (6) it follows that the fourth coefficient must be zero too and therefore we obtain Eq. (17) also for the identical bosons. Thus, using (6) again, we obtain

$$
a_{i} d_{i}=b_{i} c_{i}=a_{i} c_{i}=b_{i} d_{i}=0 .
$$

Therefore, at least two coefficients out of four, are zero: either $a_{i}=b_{i}=0$ or $c_{i}=d_{i}=0$.

Let us assume now $\alpha_{i j} \neq 0$ (and therefore $\beta_{i j}=\gamma_{i j}=$ $\left.\delta_{i j}=0\right)$ and assume that $a_{i}=b_{i}=0$. Then, equations (8) become

$$
\begin{gathered}
\alpha_{i j}=a_{j} d_{i}-b_{j} c_{i} \neq 0, \\
\beta_{i j}=a_{j} d_{i}+b_{j} c_{i}=0, \\
\gamma_{i j}=a_{j} c_{i}-b_{j} d_{i}=0, \\
\delta_{i j}=a_{j} c_{i}+b_{j} d_{i}=0 .
\end{gathered}
$$

These equations, however, do not have a solution. It is easy to see that also for all other cases there are no solutions which proves the statement for bosons.
For bosons, in contrast with the case of non-identical particles, it is possible to measure degenerate Bell operator which distinguishes two Bell states. When we consider degenerate Bell operator, it is not true anymore that at least three out of the coefficients $\alpha_{i i}, \beta_{i i}, \gamma_{i i}, \delta_{i i}$ are zero. A particular solution 8 10 allows to distinguish two out of four Bell states. The unitary linear transformation is

$$
\begin{aligned}
|\uparrow\rangle_{L} & \rightarrow \frac{1}{\sqrt{2}}(|1\rangle+|3\rangle), \\
|\downarrow\rangle_{L} & \rightarrow \frac{1}{\sqrt{2}}(|2\rangle+|4\rangle), \\
|\uparrow\rangle_{R} & \rightarrow \frac{1}{\sqrt{2}}(|1\rangle-|3\rangle), \\
|\downarrow\rangle_{R} & \rightarrow \frac{1}{\sqrt{2}}(|2\rangle-|4\rangle),
\end{aligned}
$$

and it leads to

$$
\begin{aligned}
& \left|\Psi_{-}\right\rangle \rightarrow \quad \frac{1}{\sqrt{2}}(|2\rangle|3\rangle-|1\rangle|4\rangle), \\
& \left|\Psi_{+}\right\rangle \rightarrow \quad \frac{1}{\sqrt{2}}(|1\rangle|2\rangle-|3\rangle|4\rangle), \\
& \left|\Phi_{-}\right\rangle \rightarrow \frac{1}{2}(|1\rangle|1\rangle-|3\rangle|3\rangle-|2\rangle|2\rangle+|4\rangle|4\rangle), \\
& \left|\Phi_{+}\right\rangle \rightarrow \frac{1}{2}(|1\rangle|1\rangle-|3\rangle|3\rangle+|2\rangle|2\rangle-|4\rangle|4\rangle) .
\end{aligned}
$$

(Note again that symmetrization is not written explicitly. For example, $|3\rangle|4\rangle$ means $(1 / \sqrt{2})\left(|3\rangle_{1}|4\rangle_{2}+|4\rangle_{1}|3\rangle_{2}\right)$.) This scheme can be realized in a laboratory for photon polarization states using a beam-splitter followed by polarizing beam-splitters and four detectors [11], see Fig. 1.

The most difficult case for the analysis is when the particles are identical fermions. Due to the (anti)symmetrization, Eq. (7) holds for any choice of basic unitary evolutions (3) and therefore we do not have equations analogous to (6) but only the analog of equations (8):

$$
\begin{gathered}
\alpha_{i j}=a_{i} d_{j}-a_{j} d_{i}-\left(b_{i} c_{j}-b_{j} c_{i}\right), \\
\beta_{i j}=a_{i} d_{j}-a_{j} d_{i}+b_{i} c_{j}-b_{j} c_{i}, \\
\gamma_{i j}=a_{i} c_{j}-a_{j} c_{i}-\left(b_{i} d_{j}-b_{j} d_{i}\right), \\
\delta_{i j}=a_{i} c_{j}-a_{j} c_{i}+b_{i} d_{j}-b_{j} d_{i} .
\end{gathered}
$$

Again, measurability of the nondegenerate Bell operator means that there is at least one nonzero coefficient of every kind $\alpha_{i j}, \beta_{i j}, \gamma_{i j}, \delta_{i j}$ and if, for a certain $i, j$, it is not zero, then all others are zero. Thus, we can obtain from (14) the following equations:

If the detection of $|i\rangle|j\rangle$ signifies finding $\left|\Psi_{-}\right\rangle$or $\left|\Psi_{+}\right\rangle$, i.e., $\alpha_{i j}$ or $\beta_{i j}$ are not zero, then

$$
\frac{a_{i}}{a_{j}}=\frac{c_{i}}{c_{j}} \neq \frac{b_{i}}{b_{j}}=\frac{d_{i}}{d_{j}} .
$$




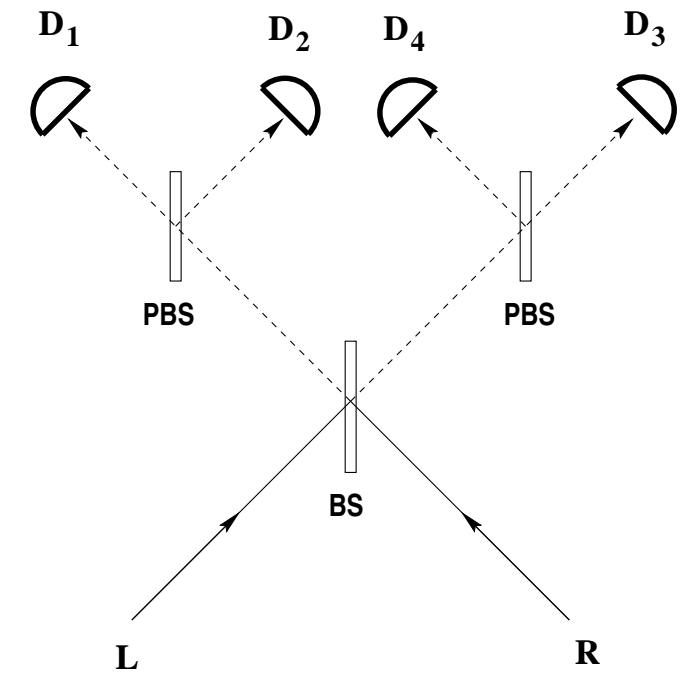

Fig. 1. A scheme for the Bell operator measurement which distinguishes two out four Bell states. The beam-splitter BS performs (up to irrelevant phases) interaction (12), where for the input states: $|\uparrow\rangle \equiv|\leftrightarrow\rangle,|\downarrow\rangle \equiv|\uparrow\rangle$, and for the output states: $|1\rangle \equiv|\leftrightarrow\rangle_{L},|2\rangle \equiv|\uparrow\rangle_{L},|3\rangle \equiv|\leftrightarrow\rangle_{R}|4\rangle \equiv|\uparrow\rangle_{R}$. The polarization beam-splitters PBS transmit horizontal polarization and reflect vertical polarization. In this configuration each detector $D_{i}$ detects the state $|i\rangle$. The outcomes $(2,3)$ and $(1,4)$ signifies detecting of $\left|\Psi_{-}\right\rangle$, while $(1,2)$ and $(3,4)$ signifies detecting of $\left|\Psi_{+}\right\rangle$. The outcomes $(1,1),(2,2),(3,3)$ and $(4,4)$ correspond both to the detection of $\left|\Phi_{-}\right\rangle$and to the detection of $\left|\Phi_{+}\right\rangle$which cannot be distinguished in this scheme.

If the detection of $|i\rangle|j\rangle$ signifies finding $\left|\Phi_{-}\right\rangle$or $\left|\Phi_{+}\right\rangle$, i.e., $\gamma_{i j}$ or $\delta_{i j}$ are not zero, then

$$
\frac{a_{i}}{a_{j}}=\frac{d_{i}}{d_{j}} \neq \frac{b_{i}}{b_{j}}=\frac{c_{i}}{c_{j}}
$$

The equations (15), (16) are valid provided there are no vanishing denominators.

Let us first prove that there cannot be a "common" state in the product states corresponding to finding $|\Psi\rangle$ (+ or - ) and $|\Phi\rangle$ (+ or -). Let us assume the opposite, that, say, $|i\rangle|j\rangle$ corresponds to finding $|\Psi\rangle$, while $|k\rangle|j\rangle$ corresponds to finding $|\Phi\rangle$. Then we have equations (15) as they are and equation (16) with index $k$ instead of $i$ :

$$
\frac{a_{k}}{a_{j}}=\frac{d_{k}}{d_{j}} \neq \frac{b_{k}}{b_{j}}=\frac{c_{k}}{c_{j}} .
$$

First, we will see that there cannot be zero in any of the denominators in equations (15) and (17), i.e., that none of the coefficients with index $j$ vanish. From the fact that for the indices $\{i, j\}$ exactly one equation out of (14) does not vanish follows that the two coefficients of the same kind, e.g., $a_{i}, a_{j}$ cannot vanish simultaneously. The same is true for the indices $\{k, j\}$. Since $|i\rangle|j\rangle$ corresponds to finding $|\Psi\rangle$ we must have $\gamma_{i j}=\delta_{i j}=0$ and $\alpha_{i j} \neq 0$ or $\beta_{i j} \neq 0$. Then, from (14) follows that vanishing coefficients might appear only in pairs: $a_{j}=c_{j}=0$ or $b_{j}=d_{j}=0$. Similarly, since $|k\rangle|j\rangle$ corresponds to finding $|\Phi\rangle$, we might have either $a_{j}=d_{j}=0$ or $b_{j}=c_{j}=0$. Therefore, if one coefficient vanishes then all coefficients vanish, which is impossible. Therefore, the denominators in equations (15) and (17) do not vanish. We can always find an equality in (15) which is not $0=0$ and we can divide corresponding inequality in (17) by the equality. We obtain

$$
\frac{a_{k}}{a_{i}} \neq \frac{c_{k}}{c_{i}} \text { or } \frac{b_{k}}{b_{i}} \neq \frac{d_{k}}{d_{i}} .
$$

Similarly, dividing the inequality from (15) by the equality from (16) leads to

$$
\frac{a_{k}}{a_{i}} \neq \frac{d_{k}}{d_{i}} \text { or } \frac{b_{k}}{b_{i}} \neq \frac{c_{k}}{c_{i}} .
$$

From equation (18) follows that $|i\rangle|k\rangle$ corresponds to finding $|\Phi\rangle$ while equation (19) yields that $|i\rangle|k\rangle$ corresponds to finding $|\Psi\rangle$. This contradiction ends the proof that it cannot be that $|i\rangle|j\rangle$ corresponds to finding $|\Psi\rangle$, while $|k\rangle|j\rangle$ corresponds to finding $|\Phi\rangle$.

We have shown that if detecting $|i\rangle|j\rangle$ corresponds to finding $|\Psi\rangle$ while detecting $|k\rangle|m\rangle$ corresponds to finding $|\Phi\rangle$ then all four states $|i\rangle,|j\rangle,|k\rangle,|m\rangle$ are different. From equation (15) and equation (17), with index $m$ instead of $j$, we always can find an equality and the inequality for the same type of coefficients. For example,

$$
\frac{a_{i}}{a_{j}}=\frac{c_{i}}{c_{j}}, \quad \frac{a_{k}}{a_{m}} \neq \frac{c_{k}}{c_{m}} .
$$

Thus, there must be at least one out of the following inequalities

$$
\frac{a_{i}}{a_{m}} \neq \frac{c_{i}}{c_{m}}, \quad \text { or } \quad \frac{a_{j}}{a_{k}} \neq \frac{c_{j}}{c_{k}} .
$$

Therefore, either $|i\rangle|m\rangle$ or $|k\rangle|j\rangle$ corresponds to detection of $|\Phi\rangle$. This contradicts what we have proved above, since $|i\rangle|j\rangle$ corresponds to detection of $|\Psi\rangle$. We have proved that also for fermions it is impossible to measure nondegenerate Bell operator without interaction between two quantum systems.

Note that nondegenerate Bell operator measurement which allows to distinguish two out of four Bell states can be performed. In fact, it can be done in the same way as with bosons. The single-particle transformations (12) leads (instead of (13) for bosons) to

$$
\begin{aligned}
& \left|\Psi_{+}\right\rangle \rightarrow \frac{1}{\sqrt{2}}(|1\rangle|2\rangle-|3\rangle|4\rangle), \\
& \left|\Psi_{-}\right\rangle \rightarrow \frac{1}{\sqrt{2}}(|3\rangle|2\rangle-|1\rangle|4\rangle), \\
& \left|\Phi_{-}\right\rangle \rightarrow \frac{1}{\sqrt{2}}(-|1\rangle|3\rangle-|2\rangle|4\rangle), \\
& \left|\Phi_{+}\right\rangle \rightarrow \frac{1}{\sqrt{2}}(-|1\rangle|3\rangle+|2\rangle|4\rangle) .
\end{aligned}
$$


(Again, the symmetrization is not written explicitly, e.g., $|3\rangle|4\rangle$ means $(1 / \sqrt{2})\left(|3\rangle_{1}|4\rangle_{2}-|4\rangle_{1}|3\rangle_{2}\right)$. We see that finding in the final measurement $|1\rangle|2\rangle$ or $|3\rangle|4\rangle$ signifies detecting of $\left|\Psi_{-}\right\rangle$while finding in $|3\rangle|2\rangle$ or $|1\rangle|4\rangle$ signifies detecting of $\left|\Psi_{+}\right\rangle$. States $|1\rangle|3\rangle$ and $|2\rangle|4\rangle$ correspond to both $\left|\Phi_{-}\right\rangle$and $\left|\Phi_{+}\right\rangle$which cannot be distinguished in this scheme. (Slight modification of this method is required for distinguishing any other two out of four Bell states.)

\section{TELEPORTATION USING INTERACTION BETWEEN QUANTUM SYSTEMS}

If we allow interaction between quantum particles we can achieve reliable ( $100 \%$ efficient teleportation). In this case we can perform measurement of the nondegenerate Bell operator, or we can use the method of "crossed" nonlocal measurements [6].

For measurement of the Bell operator consider the interaction between the particles, say, according to the following rule:

$$
\begin{aligned}
& |\uparrow\rangle|\uparrow\rangle \rightarrow|\uparrow\rangle|\downarrow\rangle, \\
& |\uparrow\rangle|\downarrow\rangle \rightarrow|\uparrow\rangle|\uparrow\rangle, \\
& |\downarrow\rangle|\uparrow\rangle \rightarrow|\downarrow\rangle|\uparrow\rangle, \\
& |\downarrow\rangle|\downarrow\rangle \rightarrow|\downarrow\rangle|\downarrow\rangle .
\end{aligned}
$$

This interaction is a "conditional spin flip". It transforms the Bell states (2) to product states

$$
\begin{aligned}
& \left|\Psi_{-}\right\rangle \rightarrow \frac{1}{\sqrt{2}}(|\uparrow\rangle-|\downarrow\rangle)|\uparrow\rangle, \\
& \left|\Psi_{+}\right\rangle \rightarrow \frac{1}{\sqrt{2}}(|\uparrow\rangle+|\downarrow\rangle)|\uparrow\rangle, \\
& \left|\Phi_{-}\right\rangle \rightarrow \frac{1}{\sqrt{2}}(|\uparrow\rangle-|\downarrow\rangle)|\downarrow\rangle, \\
& \left|\Phi_{+}\right\rangle \rightarrow \frac{1}{\sqrt{2}}(|\uparrow\rangle+|\downarrow\rangle)|\downarrow\rangle,
\end{aligned}
$$

which can be measured by local detectors. Note that the conditional spin flip (23) is equivalent (in another bases) to the conditional phase flip:

$$
\begin{gathered}
|\uparrow\rangle|\uparrow\rangle \rightarrow|\uparrow\rangle|\uparrow\rangle, \\
|\uparrow\rangle|\downarrow\rangle \rightarrow-|\uparrow\rangle|\downarrow\rangle, \\
|\downarrow\rangle|\uparrow\rangle \rightarrow|\downarrow\rangle|\uparrow\rangle, \\
|\downarrow\rangle|\downarrow\rangle \rightarrow|\downarrow\rangle|\downarrow\rangle .
\end{gathered}
$$

This is so when we use the $\hat{x}$ direction (instead of $\hat{z}$ ) for the spin of the second particle. This interaction is nonlinear in a sense that one quantum system changes its state depending on the state of another quantum system.

In a slightly different method for Bell measurement, the two particles do not interact one with the other but both interact with an auxiliary quantum particle. Consider consecutive interactions of the two particles with a spin $-\frac{1}{2}$ particle prepared initially in the state $|\uparrow\rangle$. Each interaction is described by (23) when the second terms in the products signify the spin states of the auxiliary particle. This operation allows to distinguish between $|\Psi\rangle$ and $|\Phi\rangle$ states. Indeed, each of the $|\Psi\rangle$ states leads to the flip of the spin of the auxiliary particle, while each of the $|\Phi\rangle$ states does not. In order to distinguish between $\left|\Psi_{-}\right\rangle$ and $\left|\Psi_{+}\right\rangle$(or between $\left|\Phi_{-}\right\rangle$and $\left|\Phi_{+}\right\rangle$) we have to repeat the procedure, i.e. to perform the two consecutive measurements (23) but now in the spin- $x$ component bases.

Another way to view the measurement procedure for Bell measurement described above is to consider it as consecutive measurements of two two-particle variables:

$$
\begin{aligned}
& \left(\sigma_{1 z}+\sigma_{2 z}\right) \bmod 4 \\
& \left(\sigma_{1 x}+\sigma_{2 x}\right) \bmod 4
\end{aligned}
$$

(The spin components are measured in the units of $\frac{\hbar}{2}$.) These two-particle variables are "local" since both particle 1 and particle 2 are located at the same site, but these variables can be measured even if the particles located in spatially separated sites, in which case they called nonlocal measurements [12]. A modification of nonlocal measurements when we "cross" the interactions with the two particles in time, leads to an alternative method of teleportation [6]. In order to teleport a quantum state from particle 1 to particle 2 and, at the same time, the quantum state of particle 2 to particle 1 , the following (nonlocal in space-time) variables should be measured (see Fig. $2)$ :

$$
\begin{aligned}
& \mathcal{Z} \equiv\left(\sigma_{1 z}\left(t_{1}\right)+\sigma_{2 z}\left(t_{2}\right)\right) \bmod 4 \\
& \mathcal{X} \equiv\left(\sigma_{1 x}\left(t_{2}\right)+\sigma_{2 x}\left(t_{1}\right)\right) \bmod 4
\end{aligned}
$$

For any set of outcomes of the nonlocal measurements (27) the spin state is teleported; in some cases the state is rotated by $\pi$ around one of the axes, but the resulting rotation can be inferred from the nonlocal measurements. We can complete, then, the teleportation by the following transformations

$\begin{array}{rc}(\mathcal{Z}, \mathcal{X}) & \pi \text { rotation } \\ (0,0) & y \text { axis } \\ (2,0) & x \text { axis } \\ (0,2) & z \text { axis } \\ (2,2) & \text { no rotation }\end{array}$

In order to perform nonlocal measurements (26) or (27), correlated pairs of auxiliary particles located in the sites of particle 1 and 2 are required. For example, for measuring $\left(\sigma_{1 z}+\sigma_{2 z}\right) \bmod 4$ a pair of spin- $\frac{1}{2}$ particles in a singlet state is used. The local interaction (23) in each site between the particle in and the auxiliary particle from the singlet is performed (the second "ket"s in the product signifies the state of the auxiliary particle). Then 


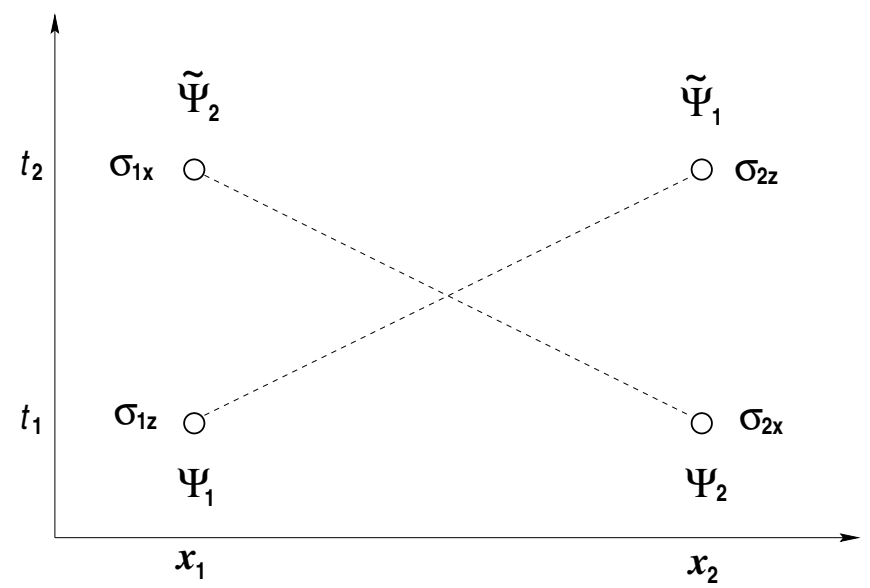

Fig. 2. Space-time diagram of "crossed" nonlocal measurements which result in two-way teleportation, i.e., swapping of the spin states. Space-time locations of local couplings are shown. When the nonlocal measurements (27) are completed, the states of the two particles are interchanged up to local $\pi$ rotations, $\tilde{\Psi}_{i}$ signifies $\Psi_{i}$ rotated according to table 28.

$\sigma_{z}$ measurement is performed on the auxiliary particles in both sites. If the outcomes are the same it means that just one auxiliary particle has been flipped, i.e., $\sigma_{1_{z}}$ and $\sigma_{2 z}$ are different and therefore $\left(\sigma_{1 z}+\sigma_{2 z}\right) \bmod 4=0$. If the outcomes are different, then either both or none of the auxiliary particles have been flipped and therefore $\left(\sigma_{1 z}+\sigma_{2 z}\right) \bmod 4=2$.

\section{TOWARDS EXPERIMENTAL REALIZATION OF RELIABLE TELEPORTATION}

In recent experiments, which announced first implementation of teleportation, the polarization state has been transported from one photon to another. The experiments performed in Innsbruck, pure state teleportation [3] and correlated state teleportation [4], have (theoretical) success rate of $25 \%$. A feasible modification of the experimental setup for the Bell-state analyzer (bringing it to the one described in Fig. 1) can increase the success rate of these experiments to $50 \%$. However, due to lack of an effective photon-photon interaction, these experiments cannot be modified to reach $100 \%$ success rate, as it was proved in Section III]. Popescu [13] has found an ingenious way to overcome this difficulty by using polarization and location degrees of freedom of the same photon. Thus, the Rome experiment [5] which implemented his idea has (theoretically) $100 \%$ success rate. Unfortunately, this method works only for transportation of the polarization state prepared on a particle which is the local member of the EPR pair constituting the quantum channel between the two sites. It is not applicable for teleportation of an unknown state of an external particle.
Thus, it seems that the most promising candidates for teleportation experiment which might have $100 \%$ success rate are proposals which involve atoms and electromagnetic cavities. First suggestions for such experiments were made shortly after publication of the original teleportation paper 14 16] and numerous modifications appeared since. The reason why implementation of these proposals seems to be feasible is that in this case "quantum-quantum" interaction between the system carrying the quantum state and a system from the EPR pair exists. A dispersive interaction (DI) of a Rydberg atom passing through a properly tuned micro-wave cavity leads to a conditional phase flip as in Eq. (25) depending on the presence of a photon in the cavity. A resonant interaction (RI- $\pi$ ) between the Rydberg atom and the cavity allows swapping of quantum states of the atom and the cavity. Thus, manipulation of the quantum state of the cavity can be achieved via manipulation of the state of the Rydberg atom. The atom state is transformed by sending it through appropriately tuned microwave zone. Moreover, the direct analog of conditional spin flip interaction (23) can be achieved through the Raman atom-cavityfield interaction [17]. No teleportation experiment has been performed yet using these methods, but it seems that the technology is not too far from this goal. About the progress in this direction one can learn from recent experiments on atom-cavity interactions 18].

Until further progress in technology it is not easy to predict which proposal will be implemented first. Assuming that resonant interactions between atoms and the cavity can be performed with a very good precision and that dispersive interaction is available with reasonable precision, the simplest way is to use the quantum channel consisting of a cavity and a Rydberg atom in a correlated state. A particular resonant interaction, RI- $\pi$, of an excited atom passing through an empty cavity,

$$
|e\rangle|0\rangle \rightarrow \frac{1}{\sqrt{2}}(|g\rangle|1\rangle+|e\rangle|0\rangle),
$$

prepares this quantum channel.

The quantum state to be teleported is the state of another Rydberg atom. The Bell measurement is then performed on this atom and the cavity. An interaction between the atom and the cavity, the conditional phase flip via dispersive interaction, DI,

$$
\begin{aligned}
|e\rangle|0\rangle & \rightarrow|e\rangle|0\rangle, \\
|e\rangle|1\rangle \rightarrow-|e\rangle|1\rangle, & \rightarrow|g\rangle|0\rangle \rightarrow|g\rangle|0\rangle, \\
|g\rangle|1\rangle & \rightarrow|g\rangle|1\rangle,
\end{aligned}
$$

disentangles the the following Bell states:

$$
\begin{aligned}
\left|\Psi_{ \pm}\right\rangle & =\frac{1}{2}(|e\rangle(|0\rangle-|1\rangle) \pm|g\rangle(|1\rangle+|0\rangle) \\
\left|\Phi_{ \pm}\right\rangle & =\frac{1}{2}(|e\rangle(|0\rangle+|1\rangle) \pm|g\rangle(|1\rangle-|0\rangle)
\end{aligned}
$$


The Bell states (31) have the form of Eq. 2 when the first $|\uparrow\rangle$ in the product is identified with $|e\rangle$, the second $|\uparrow\rangle$, with $(1 / \sqrt{2})(|0\rangle+|1\rangle)$, etc. Measurement of the atom state and the cavity state completes the Bell measurement procedure.

In order to make the measurement of the cavity state we perform another resonant interaction, RI- $\pi / 2$, between the cavity an auxiliary atom prepared initially in the ground state,

$$
\begin{aligned}
& |g\rangle|1\rangle \rightarrow|e\rangle|0\rangle, \\
& |g\rangle|0\rangle \rightarrow|g\rangle|0\rangle .
\end{aligned}
$$

This interaction transfers the quantum state of the cavity to this atom. The final measurements on the atoms distinguish between the states

$$
\frac{1}{\sqrt{2}}(|g\rangle+|e\rangle), \quad \frac{1}{\sqrt{2}}(|g\rangle-|e\rangle) .
$$

Since detectors can distinguish between $|g\rangle$ and $|e\rangle$, the atoms should rotate their states passing through the appropriate microwave zones before the detection. When the Bell measurement is completed, the quantum state is teleported up to the known local transformation determined by the results of the Bell measurement. The scheme for this teleportation procedure, apart from the final local transformation, is presented in Fig. 3.

Another proposal for teleportation involving single cavity [17] is based on Raman interaction. This scheme does not require microwave zone interactions, but it requires additional atom for preparing the initial state of the cavity, $(1 / \sqrt{2})(|0\rangle+|1\rangle)$. A very recent single-cavity teleportation proposal [19], which employs three-particle (cavity and two atoms) correlated state, seems to be more complicated. The main advantage of this scheme, that it does not require measurement of the nondegenerate Bell operator is not very important since quantumquantum interactions are available and, consequently, the Bell measurement is not too problematic. On the other hand, the complications related to the preparation of the three-particle entanglement are significant.

One of the relatively simple methods for "two-way" teleportation, i.e., swapping of quantum states of two separated atoms, is a direct implementation of crossed nonlocal-measurement procedure presented in the previous section. Two pairs of correlated micro-wave cavities are prepared as the two quantum channels. Preparation of such a channel requires consecutive resonant interaction of an auxiliary atom, prepared in the excited state: first, RI- $\pi / 2$ with the cavity in one site and second, RI- $\pi$ with the cavity in the other site, see Fig. 4a.

In the second step, the atom in each site passes, interacting dispersively, through the cavities in the "crossed" order, see Fig. 4b. The dispersive interaction is given by Eq. (30) and it corresponds to measurement of $\sigma_{z}$, because $\left|\uparrow_{z}\right\rangle$ flips the states of the cavity defined as

$$
|\uparrow\rangle \equiv \frac{1}{\sqrt{2}}(|0\rangle+|1\rangle), \quad|\downarrow\rangle \equiv \frac{1}{\sqrt{2}}(|0\rangle-|1\rangle)
$$

This is the basis of the Bell states (31). Since we also have to make coupling to $\sigma_{x}$ we have to rotate the state of the atom such that " $x$ " rotates to " $z$ " before coupling with the cavity and rotate it back after the interaction.

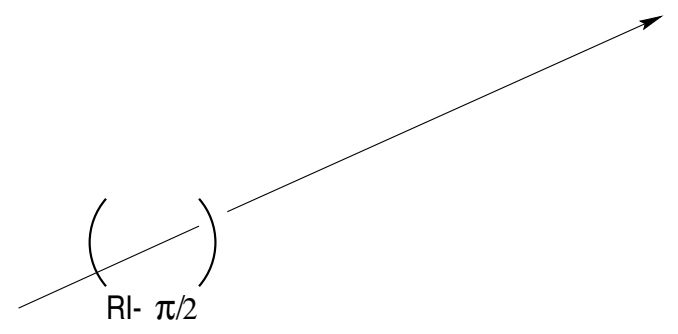

(a)

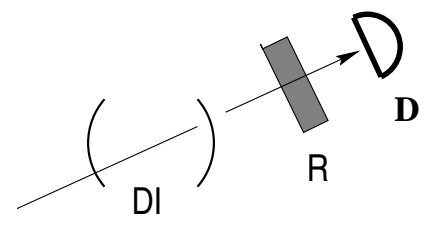

(b)

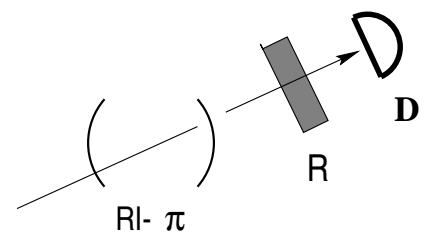

(c)

Fig. 3. Single-cavity teleportation of a quantum state of an atom. The final stage, the rotation of the state according to the results of the Bell measurement is not shown.

(a) Preparation of the quantum channel. An atom passes through a cavity and sent to a remote site. Resonant interaction RI- $\pi / 2$ given by (29) of the atom in the cavity creates the correlation.

(b) The atom, carrying the quantum state to be teleported, passes through the cavity, microwave field zone $\mathrm{R}$ and is detected by the detector D. Dispersive atom-cavity interaction DI given by (30) disentangles the Bell states (31). Microwave field zone $\mathrm{R}$ rotates the atom states (33) to $|g\rangle$ and $|e\rangle$ accordingly, which are then distinguished by the detector D.

(c) An auxiliary atom passes through the cavity in order to measure its state. Resonant interaction RI- $\pi$ transfers the states of the cavity to the atom. Then, the atom states are rotated in the microwave zone $\mathrm{R}$ and distinguished by the detector $\mathrm{D}$. 


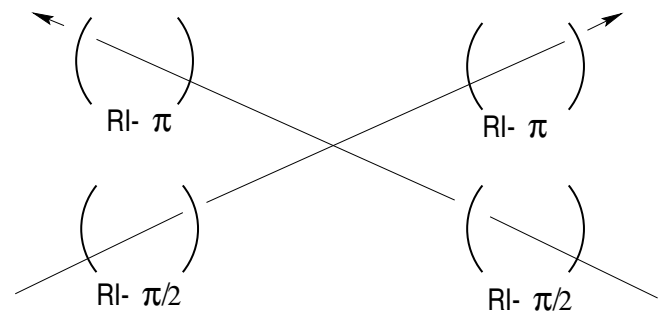

(a)

(b)
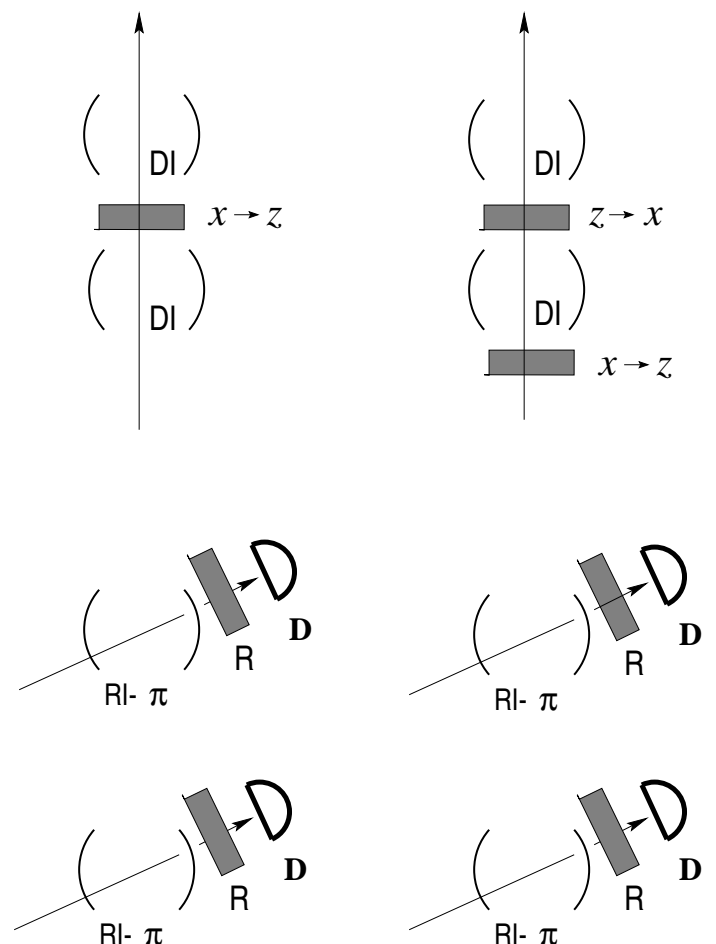

(c)

Fig. 4. Two-way teleportation of quantum states of atoms. The final rotations of the states of the atoms according to the results of nonlocal measurements are not shown.

(a) Preparation of the quantum channels. Atom in the excited state makes resonant interaction RI- $\pi / 2$ with a cavity in one site and resonant interaction RI- $\pi$ with corresponding cavity in another site. Another atom makes the same interactions with the second pair of cavities. Each pair ends up in a correlated state $(1 / \sqrt{2})(|0\rangle|1\rangle+|1\rangle|0\rangle)$.

(b) Coupling of the atoms with the quantum channels. An atom on each site passes through two cavities making dispersive interactions DI. In between, the atoms pass through microwave zones which make the appropriate rotations of their quantum states.

(c) Local measurements of the quantum states of the cavities which yield the results of the nonlocal measurements. Auxiliary atoms pass through cavities in order to measure their states. Resonant interactions RI- $\pi$ transfer the states of the cavities to the atoms. Then, the atom states are rotated in the microwave zones $\mathrm{R}$ and distinguished by detectors $\mathrm{D}$.
The third step, described in Fig. 4c, is the measurement of the states of the cavities in the basis (34). This stage, requires an auxiliary atom for each cavity. The resonant interaction transfers the correlated state of the pairs of cavities to the correlated states of the atoms. Then the atoms pass through microwave zones rotating their states before detection by the detectors which distinguish between ground and excited states. These outcomes of these local measurements yield the result of the nonlocal measurements (27) which determine the final transformation to be performed on the atoms (to be combined with $z \rightarrow x$ rotation for the first atom which was left out, see Fig. 4b.). Completing all stages of the procedure described above results in two-way teleportation.

Note another (seemingly less economical) proposal for a two-way teleportation of atom states using interaction with cavities 20]. It essentially doubles one of the original atom -cavity teleportation schemes 15 reusing atoms which bring the quantum states for receiving the quantum states from the other site after "stripping" from them quantum information. Anyway, today the two-way teleportation is still a gedanken experiment, at least until one-way teleportation will be implemented.

One difficulty with teleportation of atomic states which should be mentioned is that usual experiments are performed with atomic beams and not with individual atoms. Such experiments might be good for demonstration and studying experimental difficulties for teleportation, but they cannot be considered as implementation of the original wisdom of teleportation or used for cryptographic purposes. In fact, optical experiments have this difficulty too, unless "single-photon" guns will be used. Both for atoms and in the optical regime this problem does not seem to be unsolvable, but it certainly brings attention to experiments with trapped ions, the experiments which individual quantum systems. There are many similarities between available manipulations with atoms and with ions so the methods discussed above might be implemented for ion systems too.

\section{TELEPORTATION OF CONTINUOUS VARIABLES}

In the framework of nonlocal measurements there is a natural way of extending the teleportation scheme to the systems with continuous variables [6]. Consider two similar systems located far away from each other and described by continuous variables $q_{1}$ and $q_{2}$ with corresponding conjugate momenta $p_{1}$ and $p_{2}$. In order to teleport the quantum state of the first particle $\Psi_{1}\left(q_{1}\right)$ to the second particle (and the state of the second particle $\Psi_{2}\left(q_{2}\right)$ to the first) we perform the following "crossed" nonlocal measurements (see Fig. 5), obtaining the outcomes $a$ and $b$ : 


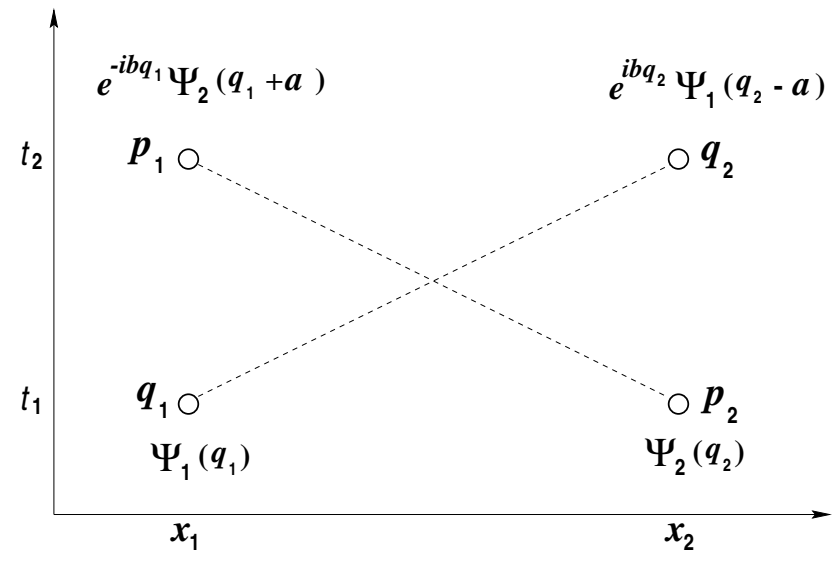

Fig. 5. Space-time diagram of "crossed" nonlocal measurements which result in two-way teleportation of quantum states of quantum systems with continuous variables. Spacetime locations of local couplings are shown. When the nonlocal measurements (35) are completed, the states of the two particles are interchanged up to the known shifts in $q$ and $p$.

$$
\begin{aligned}
& q_{1}\left(t_{1}\right)-q_{2}\left(t_{2}\right)=a, \\
& p_{1}\left(t_{2}\right)-p_{2}\left(t_{1}\right)=b .
\end{aligned}
$$

In Ref. [6] it is shown that these nonlocal "crossed" measurements "swap" the quantum states of the two particles up to the known shifts in $q$ and $p$. Indeed, the states of the particles after completion of the measurements 35 are

$$
\begin{gathered}
\Psi_{f}\left(q_{1}\right)=e^{i b q_{1}} \Psi_{2}\left(q_{1}+a\right), \\
\Psi_{f}\left(q_{2}\right)=e^{-i b q_{2}} \Psi_{1}\left(q_{1}-a\right) .
\end{gathered}
$$

The state of particle 2 after $t_{2}$, is the initial state of the particle 1 shifted by $-a$ in $q$ and by $-b$ in $p$. Similarly, the state of particle 1 is the initial state of particle 2 shifted by $a$ in $q$ and by $b$ in $p$. After transmitting the results of the local measurements, $a$ and $b$, the shifts can be corrected by appropriate kicks and back shifts (even if the quantum state is unknown), thus completing a reliable teleportation of the state $\Psi_{1}\left(q_{1}\right)$ to $\Psi_{1}\left(q_{2}\right)$ and of the state $\Psi_{2}\left(q_{2}\right)$ to $\Psi_{2}\left(q_{1}\right)$.

In order to perform nonlocal measurements of continuous variables (35) a quantum channel is required. While for the case of spin- $\frac{1}{2}$ particles the quantum channel was an $\operatorname{EPR}(-B o h m)$ pair, for continuous variables the entangled state of the original EPR paper is required:

$$
\begin{gathered}
Q_{1}+Q_{2}=0, \\
P_{1}-P_{2}=0,
\end{gathered}
$$

where $Q_{1}$ and $Q_{2}$ are continuous variables of the pair of auxiliary particles with corresponding conjugate momenta $P_{1}$ and $P_{2}$. Two local von Neumann type interactions which can be written in the interaction Hamiltonian

$$
H=g\left(t-t_{1}\right) P_{1} q_{1}-g\left(t-t_{2}\right) P_{2} q_{2},
$$

with normalized and localized around zero $g(t)$ lead to final state of auxiliary particles such that

$$
Q_{1 f}+Q_{2 f}=q_{1}\left(t_{1}\right)-q_{2}\left(t_{2}\right) .
$$

Thus, local measurements of $Q_{1 f}$ and $Q_{2 f}$ yield $a$ (of Eq. (35)). Another EPR pair is needed for measuring $b$.

A generalization of the BBCJPW scheme to the case of continuous variables is also possible. The quantum channel is again the EPR state (37). Now, an analog of Bell measurement for the systems with variables $q$ which carries the quantum state and the auxiliary system with variable $Q_{1}$ is needed. While it is not easy to make generalization of the Bell operator through definition of its eigenstates, it is straightforward to generalize Bell operator measurement from the two consecutive nonlocal measurements (26) applied in the spin- $\frac{1}{2}$ particles case, to the following two consecutive nonlocal measurements

$$
\begin{gathered}
q+Q_{1}, \\
p-P_{1} .
\end{gathered}
$$

These measurements ends up in finding one of the "shifted" EPR states, where the shifts are the outcomes of the measurements:

$$
\begin{gathered}
q+Q_{1}=a \\
p-P_{1}=b .
\end{gathered}
$$

At the end of this measurement the quantum state $\Psi(q)$ is teleported, again up to the known shifts, to the remote particle of the EPR pair, the state of which becomes

$$
\Psi_{f}\left(Q_{2}\right)=e^{i b Q_{2}} \Psi\left(Q_{2}+a\right) .
$$

The final stage of this teleportation procedure consist of the appropriate back shifts of the state in $Q_{2}$ and $P_{2}$ which result in transporting the quantum state $\Psi(q)$ in site 1 to the same quantum state $\Psi\left(Q_{2}\right)$ of a system in site 2 . Of course, this method, unlike the crossed measurements scheme, yields only one-way teleportation. Both methods transport not just pure states but also correlations, when the input particle is not in a pure state before teleportation.

Surprisingly, reliable teleportation of continuous variables seems to be possible to implement in a real laboratory. Braunstein and Kimble made a realistic proposal for teleporting quantum state of a single mode of the electro-magnetic field 21. This remarkable result is an implementation of the scheme described in the previous paragraph. In their methods $q$ is " $x$ " defined for a single mode of an elector-magnetic field, and correspondingly $p$ is the conjugate momentum of $x$. The analog of the EPR state (37) is obtained by shining squeezed light with certain $x$ from one side and squeezed light with certain $p$ 
from the other side of a simple beam splitter. The analog of measurements (40) is achieved using another simple beam splitter and homodyne detectors. The shifts in $x$ and $p$ which complete the teleportation procedure can be done by combining the output field with coherent state of an appropriate amplitudes fixed by the results of the homodyne measurements. Preliminary experimental results for such teleportation procedure has been obtained 22. Note also a very recent proposal [23] for teleporting of a single-photon wave packet.

\section{CONCLUSIONS}

We have shown that without "quantum-quantum" interaction one cannot perform complete measurement of Bell nondegenerate operator and therefore, one cannot perform reliable teleportation of photon polarization states using method employed in recent experiments, i.e., experiments without quantum-quantum interactions. Therefore, another methods for teleportation have to be developed. Today's technology allows quantum-quantum interaction between atoms and electro-magnetic field in cavities which makes teleportation schemes using such elements good candidates for implementation reliable teleportation. Various schemes are briefly analyzed, in particular, using the language of nonlocal measurement which proved to be helpful for such problems. Schemes for one- and two-way teleportation which seems to be easiest for implementation are proposed.

We have discussed methods for teleportation of system described by continuous variables. One may see an apparent contradiction between the proof of Section III that $100 \%$ efficient teleportation cannot be achieved using linear elements and single-particle state detectors and the result of Braunstein and Kimble presented at the end of the last section according to which one can perform a reliable teleportation of quantum state of a system with continuous variables using beam-splitters and local measuring devices. Indeed, it is natural to assume that if reliable teleportation of a quantum state of a two-level system is impossible at certain circumstances, it is certainly impossible for quantum states of systems with continuous variables. However, although it is not immediately obvious, the circumstances are very different. There are several differences. The analog of Bell operator for continuous variable does not have among its eigenvalues four states of the general form (2) where $|\uparrow\rangle$ and $|\downarrow\rangle$ signify some orthogonal states. Even more importantly, the beam-splitter, a simple half-silvered mirror is not a linear element in the sense of Section III. If we do not send any "light" from one side of the beamsplitter, we still get the vacuum field from this port. The beam-splitter in Braunstein-Kimble experiment leads to "quantum-quantum" interaction: the variable $x$ of one of the output ports of the beam splitter becomes equal to $\frac{1}{\sqrt{2}}\left(x_{1}+x_{2}\right)$, essentially, the sum of the quantum variables of the input port. The absence of such "quantumquantum" interactions is an essential ingredient in the proof of Section III. The beam-splitter, however, is linear for photons, but the homodyne detectors which measure $x$ are not single-particle detectors for photons - using single-particle measuring devices is another constraint used in the proof. The Braunstein-Kimble method is not applicable directly for teleporting $\Psi(x)$ where $x$ is a spatial position of a quantum system. Additional quantumquantum interaction which converts continuous variable of a real particle to the abstract (although measurable) variable $x$ of their method is required.

The fact that Braunstein-Kimble method does not contradict the proof does not make the method less interesting. The task is to find feasible proposals for teleportation. The reason why linear devices were considered in the proof is because usually it is more difficult to implement nonlinear interactions in a laboratory. Half-silvered mirror, even if it can be considered as a nonlinear device in some sense, can be easily used in a laboratory. (The main experimental difficulty of the Braunstein-Kimble proposal is preparation of highly squeezed light, which is necessary for high-efficiency teleportation.) On the other hand, the fact that there are realistic proposals for teleportation which do not fulfill the assumption of the proof of Section III does not make the proof irrelevant. It still limits wide class of teleportation proposals, in particular those in which quantum states are encoded in photon polarization or photon location.

Although it is argued in this paper that current teleportation experiments cannot lead to reliable teleportation procedure and that for alternative proposals some technological tools are currently not available, we are optimistic about solution of the problem in a foreseen future. Much efforts made in this direction because teleportation and related experiments are building blocks of quantum cryptography and quantum computation: the two extremely important fields which are on the verge of transformation from gedanken ideas to reality.

The research was supported in part by grant $614 / 95$ of the Basic Research Foundation (administered by the Israel Academy of Sciences and Humanities). Part of this work was done during the 1998 Elsag-Bailey Foundation research meeting on quantum computation.

[1] The Oxford English Dictionary, 2nd Ed. (Clarendon Press, Oxford 1989) XVII p. 730.

[2] C. H. Bennett, G. Brassard, C. Crepeau, R. Jozsa, A. Peres, W. K. Wootters, Phys. Rev. Lett. 70, 1895 (1993). 
[3] B. Bouwmeester, J. W. Pan, K. Mattle, M. Eibl, H. Weinfurter, A. Zeilinger, Nature 390, 575 (1997).

[4] J. W. Pan, D. Bouwmeester, H. Weinfurter, and A. Zeilinger Phys. Rev. Lett. 80, 3891 (1998).

[5] D. Boschi, S. Branca, F. De Martini, L. Hardy and S. Popescu, Phys. Rev. Lett. 80, 1121 (1997).

[6] L.Vaidman, Phys. Rev. A 49, 1473 (1994).

[7] Before completing the manuscript we have learned that J. Calsamiglia and N. Lütkenhaus (unpublished) considered independently this proof for photons. They also proved impossibility of the nondegenerate Bell measurement for the case which is not considered here. They allowed indirect interaction between quantum particles by making measurements in two stages such that the choice of the measurements in the second stage depends on the results of the measurements in the first stage.

[8] H. Weinfurter, Europhys. Lett. 559 (1994).

[9] S. L. Braunstein and A. Mann, Phys. Rev. A 51, R1727 (1955).

[10] M. Michler, K. Mattle, H. Weinfurter, and A. Zeilinger, Phys Rev. A 53, R1209 (1996).

[11] K. Mattle, M. Eibl, H. Weinfurter, A. Zeilinger, in Quan- tum Interferometry, F. De Martini, G. Denardo and Y. Shih, eds., (VCH, Weinheim 1996) p. 57.

[12] Y. Aharonov, D. Albert, and L.Vaidman, Phys. Rev. D 34, 1805 (1986).

[13] S. Popescu, e-print quant-ph/9501020.

[14] T. Sleator and H. Weinfurter, in IQEC Technical Digest 1994, Vol. 9, 1994, OSA Technical Digest Series (OSA, Washington, D.C., 1994), p. 140.

[15] L. Davidovich, N. Zagury, M. Brune, J. M. Raimond and S. Haroche, Phys. Rev. A, 50, 895, (1994).

[16] J. I. Cirac and A. S. Parkins, Phys. Rev. A 50 R4441 (1994).

[17] S. B. Zheng, G. C. Guo, Phys. Lett. A 232, 171 (1997).

[18] S. Haroche, M. Brune, J. R. Raimond, Philos. T. Roy. Soc. A 355 (1733) 2367 (1997).

[19] N. G. Almeida, L. P. Maia, C. J. Villas-Boas, M. H. Y. Moussa, Phys. Lett. A 241, 213 (1998).

[20] M. H. Y. Moussa, Phys. Rev. A 55 R3287 (1997).

[21] S. Braunstein, H. J. Kimble, Phys. Rev. Lett. 80, 869 (1998).

[22] S. Braunstein, talk at Benasque Center of Physics.

[23] S. N. Molotkov, Phys. Lett. A 245, 339 (1998). 